\title{
Philosophiques
}

\section{Quelques aspects de la première théorie du jugement de Husserl}

\section{Robin D. Rollinger}

Volume 36, numéro 2, automne 2009

Edmund Husserl (1859-1938)

URI : https://id.erudit.org/iderudit/039477ar

DOI : https://doi.org/10.7202/039477ar

Aller au sommaire du numéro

\section{Éditeur(s)}

Société de philosophie du Québec

ISSN

0316-2923 (imprimé)

1492-1391 (numérique)

Découvrir la revue

Citer cet article

Rollinger, R. D. (2009). Quelques aspects de la première théorie du jugement de Husserl. Philosophiques, 36(2), 381-398. https://doi.org/10.7202/039477ar

\section{Résumé de l'article}

La théorie du jugement était une des préoccupations de Husserl depuis la toute première période de sa carrière. Ses premières recherches dans ce domaine se trouvent dans deux manuscrits rédigés en 1893 et 1893-1894 et publiés dans le volume XL des Husserliana (Texte $\mathrm{n}^{0} 1$ et 2). Dans cet article, j'examinerai la théorie du jugement dans ces manuscrits en relation aux questions suivantes : 1) les jugements en relation aux représentations ; 2) les assomptions comme des actes qui se déroulent parallèlement aux jugements ; 3 ) les jugements impropres en tant que distincts des jugements propres ; 4) les jugements objectifs ; 5) les états de choses en tant que corrélatifs des actes de juger. Nous verrons que, tandis que Husserl se libère à maints égards de la théorie du jugement de Brentano qu'il avait apprise à Vienne puis (de Stumpf) à Halle, ses positions sont en même temps tout à fait représentatives de la phénoménologie autrichienne dans la mesure où elles impliquent à la fois une psychologie descriptive et une théorie de l'objet, bien que sans aucune prétention d'établir une nouvelle méthode en philosophie. 


\title{
Quelques aspects de la première théorie du jugement de Husserl
}

\author{
ROBIN D. ROLLINGER \\ Université de Salzburg
}

\begin{abstract}
RÉSUMÉ. - La théorie du jugement était une des préoccupations de Husserl depuis la toute première période de sa carrière. Ses premières recherches dans ce domaine se trouvent dans deux manuscrits rédigés en 1893 et 1893-1894 et publiés dans le volume XL des Husserliana (Texte $n^{\circ} 1$ et 2). Dans cet article, j'examinerai la théorie du jugement dans ces manuscrits en relation aux questions suivantes: 1) les jugements en relation aux représentations; 2 ) les assomptions comme des actes qui se déroulent parallèlement aux jugements; 3 ) les jugements impropres en tant que distincts des jugements propres; 4) les jugements objectifs; 5) les états de choses en tant que corrélatifs des actes de juger. Nous verrons que, tandis que Husserl se libère à maints égards de la théorie du jugement de Brentano qu'il avait apprise à Vienne puis (de Stumpf) à Halle, ses positions sont en même temps tout à fait représentatives de la phénoménologie autrichienne dans la mesure où elles impliquent à la fois une psychologie descriptive et une théorie de l'objet, bien que sans aucune prétention d'établir une nouvelle méthode en philosophie.
\end{abstract}

\begin{abstract}
The theory of judgment was one of Husserl's concerns from a very early period of his career onward. His early investigations in this area are to be found in two manuscripts which he wrote in 1893 and 1893/94, which have been published as Text Nr. 1 and Text Nr. 2 of Husserliana XL. In this paper I examine the theory of judgment in these manuscripts with regard to the issues of 1 ) judgments in relation to presentations, 2) assumptions as acts which run parallel to judgments, 3) improper judgments as distinct from improper ones, 4) objective judgments, and 5) states of affairs as correlatives of judging acts. While we see Husserl freeing himself in many respects from the Brentanian theory of judgment which he had learned in Vienna and again (from Stumpf) in Halle, it is at the same time seen that Husserl's views are quite representative of Austrian phenomenology insofar as they involve both descriptive psychology and object-theory, though without any pretense of establishing a novel method of philosophy.
\end{abstract}

\section{Introduction}

Durant le dix-neuvième siècle, et particulièrement au cours des dernières décennies de celui-ci, presque tous les philosophes engagés dans des recherches en logique, en épistémologie ou en psychologie ont formulé et défendu une théorie du jugement, qu'elle soit reprise d'autres philosophes ou qu'elle soit plutôt originale de nature ${ }^{1}$. Ce fut le cas tout particulièrement dans

1. Les trois branches de la philosophie mentionnées ici étaient inextricablement liées à l'époque. En ce qui concerne les ouvrages des philosophes importants chez qui on retrouve une théorie du jugement, voir Bolzano (1837) III; Lotze (1880); Drobisch (1887); Sigwart (1889); 
l'école de Franz Brentano², la théorie du jugement constituant la pierre de touche de la logique et de l'épistémologie brentanienne ainsi qu'un aspect crucial de sa psychologie et de son éthique ${ }^{3}$. Évidemment, cela ne signifie aucunement que les remarquables étudiants de Brentano auraient simplement adopté sa théorie, mais il leur inculqua l'importance de développer une théorie du jugement dans les recherches portant sur des sujets philosophiques majeurs. Chez Husserl tout particulièrement, qui fut sous la direction immédiate de Brentano de 1884 à 1886 à Vienne ${ }^{4}$, et ensuite sous sa direction médiate à Halle par le biais d'un de ses anciens étudiants, Carl Stumpf ${ }^{5}$, cet enseignement laissa ses traces. Pendant les années 1890, Husserl tourna son attention de la philosophie des mathématiques vers la logique et commença conséquemment à s'intéresser de plus en plus à la théorie du jugement. Bien que les conceptions du dernier Husserl sur le jugement aient été largement diffusées depuis longtemps ${ }^{6}$, le point de vue du premier Husserl sur cette question reste encore à explorer. Dans ce qui suit, je vais discuter de la théorie du jugement de Husserl en mettant l'accent sur les textes récemment publiés des années 1893 et 1894, tout particulièrement sur le Versuch über den Ursprung der Begriffe "Notwendigkeit" [und] "notwendige Folge”, über hypothetisches und kausales Urteil de 1893 et sur Eigentliche und uneigentliche Urteile (ainsi que ses appendices) de 1893-1894, qui ont été écrits avant que Husserl en vienne à considérer que les propositions

Sigwart (1893); Wundt (1893); Wundt (1894) et Bergmann (1895). Voir également les ouvrages recensés par Husserl dans Husserliana XXII, pp. 124-151 et 162-258.

2. Voir Rollinger (2004b) ou sa version révisée dans Rollinger (2008), pp. 233-261.

3. La source principale de cette théorie du jugement a longtemps été Brentano (1874), pp. 266-305, qui contient seulement une application succinte de sa théorie à la logique. Brentano a développé sa théorie du jugement plus en détail dans ses leçons de logique, particulièrement dans un cours qu'il a donné d'abord à Würzburg puis à Vienne sur le sujet (Brentano EL 80), cours qui malheureusement a été édité de manière très insatisfaisante (couplé avec des textes plus tardifs) dans Brentano (dir. Mayer-Hillebrand) (1956). Dans les archives littéraires de Brentano, on peut trouver les notes complètes d'un autre cours de logique, datant de 18841885 (Brentano EL 72), mais ces notes n'élaborent pas autant sur la théorie du jugement. Bien entendu, l'épistémologie de Brentano est entretissée avec sa logique qu'il caractérise d' "art de juger" (Kunst des Urteilens). En ce qui concerne l'application de cette théorie à l'éthique, voir Brentano (1889).

4. Pour une discussion de la relation de Husserl à Brentano, voir Rollinger (1999), pp. 18-67 ainsi que Rollinger (2004a).

5. Pour une discussion de la relation de Husserl à Stumpf, voir Rollinger (1999), pp. 83-123.

6. Cela est dû en premier lieu à la publication de Husserl (Landgrebe, dir.) (1948).

7. Husserliana XL, pp. 1-30. Ce texte sera dorénavant abrégé sous le nom de Versuch.

8. Husserliana XL, pp. 31-50. Les appendices à ce texte sont publiés dans Husserliana XL, pp. 51-67. Husserl a placé les manuscrits dont ces textes sont tirés dans le même dossier que le manuscrit d'Eigentliche und uneigentliche Urteile. Ils ont été rédigés à peu près au même moment et conçus comme des tentatives d'élaborer différents thèmes dans ce texte. 
(Sätze), et non les jugements, doivent être inclus dans le domaine d'investigation de la logique ${ }^{9}$.

\section{Représentations}

Avant d'examiner la théorie du jugement telle qu'elle est mise de l'avant dans les textes que nous venons de mentionner, il importe de glisser d'abord un mot sur le concept de représentation défendu par Husserl pendant cette même période ${ }^{10}$. Bien entendu, le concept de représentation a une longue histoire. Toutefois, en ce qui concerne l'usage qu'en fait Husserl, son point de départ se trouve dans la psychologie descriptive de Brentano ainsi que dans certains travaux des autres étudiants de Brentano ${ }^{11}$.

Même si Husserl en vint à conclure que Brentano avait tort de concevoir les jugements comme fondés sur de simples représentations (bloße Vorstellungen $)^{12}$, ses Psychologische Studien zur elementaren Logik ${ }^{13}$ commencèrent néanmoins par considérer d'abord les représentations avant même d'aborder les autres aspects de la vie consciente ${ }^{14}$. Nous nous intéressons ici à la conception husserlienne des représentations telle qu'exposée dans les Psychologische Studien (publiées pour la première fois en 1894) ${ }^{15}$ ainsi que dans un plus long texte de 1893, publié de manière posthume et étroitement lié à celles-ci ${ }^{16}$.

9. Voir Rollinger (2003) ou encore sa version révisée dans Rollinger (2008), pp. 87-108.

10. Pour une discussion plus élaborée de ces premières conceptions, voir Schuhmann (2004), pp. 101-135 et 261-277.

11. Voir par exemple Stumpf (1873), Meinong (1877), Meinong (1882) et Twardowski (1894).

12. Voir Rollinger (1999), pp. 52-56.

13. Husserliana XXII, pp. 92-123.

14. Bien que l'étude sur les représentations soit en fait la seconde de ces études, la première, qui touche à la distinction entre représentations "concrètes" et "abstraites" (Husserliana XXII, pp. 92-100) appartient davantage au domaine de la théorie de l'objet qu'à celui de la psychologie descriptive. Cette étude est effectivement un prototype de la troisième des Recherches logiques qui est d'ailleurs conçue comme une contribution à "la théorie pure (apriorique) des objets comme tels» (der reinen (apriorischen) Theorie der Gegenstände als solcher) (Husserliana XIX/1, p. 227). Comme je le note dans les remarques finales du présent article, la psychologie descriptive et la théorie de l'objet forment ensemble une unité en tant qu'elles sont les deux branches d'une seule discipline dont Husserl était un des pionniers.

15. La dernière phrase de ce texte (Husserliana XXII, p. 123) indique pleinement qu'au moins une autre étude devait suivre. De plus, la phrase «Ne peut rendre justice aux faits aucune théorie du jugement qui ne s'appuie sur une étude approfondie des relations descriptives et génétiques des intuitions et des représentations" (Dass keine Urteilstheorie den Tatsachen gerecht zu werden vermag, die sich nicht auf ein tieferes Studium der deskriptiven und genetischen Verhältnisse von Anschaunngen und Repräsentation stützt) (Husserliana XII) suggère que Eigentliche und uneigentliche Urteile, dans lequel ces sujets sont centraux, était l'esquisse d'une troisième Psychologische Studie zur elementaren Logik.

16. Husserliana XXII, pp. 269-302. Voir également les appendices dans le même volume, pp. 406-431. 
De la façon dont Brentano traite des représentations, elles sont à distinguer de manière très nette des jugements ainsi que des perceptions. Mais, en même temps, le concept est construit si amplement qu'il inclut également les actes d'imagination et les abstractions. Parmi les différents types de représentations, Brentano distingue entre les représentations simples et les représentations complexes, entre les représentations individuelles et les représentations universelles, et entre les représentations propres et impropres. Du point de vue de Brentano, nos représentations des très grands nombres et de Dieu sont des représentations impropres (uneigentlich) $)^{17}$. Dans certains cas, nous avons de telles représentations parce qu'elles sont plus pratiques que les représentations propres, alors que dans d'autres cas nous n'avons tout simplement pas de représentation propre correspondante. C'est uniquement au moyen de représentations impropres que nous pouvons avoir un concept de Dieu. C'est bien sûr ici qu'on retrouve les analogies. De plus, les expressions linguistiques et d'autres moyens symboliques ont l'avantage de nous aider à former des représentations de ce genre.

La distinction entre les représentations propres et les représentations impropres était bien sûr une distinction familière dans l'école de Brentano. Elle a été explicitement reconnue par Stumpf ${ }^{18}$, qui était très près de Brentano, ainsi que par Marty qui était encore plus près de lui ${ }^{19}$. Dans le cas de Meinong, qui était plus distant de Brentano et qui, déjà tôt dans sa carrière, suscita des critiques de sa part ${ }^{20}$, sa distinction entre représentations directes et représentations indirectes était aux yeux de Husserl simplement une terminologie différente pour dire la même chose ${ }^{21}$.

17. Voir les citations tirées de Brentano EL 80 dans Rollinger (2008), p. 34n.

18. Rollinger (1999), p. 301.

19. Marty (1884), pp. 66-70. À ce point de vue, en opposition à certains de ses contemporains, la thèse de Marty est que ce ne sont pas tous les types de penser qui sont symboliques, c.-à-d. impropres. La caractéristique importante de l'école de Brentano est donc non seulement la reconnaissance de représentations impropres, mais la distinction entre celles-ci et les représentations propres. De plus, la thèse martienne d'un penser propre est très apparentée à la conception husserlienne des concepts et des intuitions que nous abordons plus loin.

20. Voir Rollinger (2005) ou sa version révisée dans Rollinger (2008), pp. 157-188.

21. Voir Meinong (1882), pp. 92 sq. Toutefois, Brentano affirme clairement qu'il considère inacceptable la notion meinongienne de représentation indirecte comme moyen de décrire la nature des représentations impropres. Voir Rollinger (2008), p. 162. De plus, dans une lettre à Husserl (datée du 19 juin 1891), en réponse à l'affirmation faite dans le premier livre de Husserl, selon laquelle, dans ses leçons, Brentano a toujours (von jeher) fait la distinction entre représentations propres et représentations impropres (Husserliana XII, p. 193sq.), Meinong rétorque que cela ne se laisse pas appuyer sur sa propre expérience (Schuhmann (dir.), 1994, p. 129) et indique ainsi qu'il n'a pas pris sa distinction entre représentations directes et représentations indirectes de Brentano. Cela n'a toutefois pas empêché Husserl d'affirmer que «des termes comme "inadéquat", “impropre”, "représentations indirectes” signifient des Repräsentationen et les termes opposés correspondants signifient des intuitions» (Husserliana XXII, p. 119). 
Comme ses tout premiers intérêts philosophiques touchèrent la philosophie des mathématiques, et particulièrement la philosophie de l'arithmétique $^{22}$, Husserl fit usage de la notion de représentation impropre dans ses recherches de cette période et se référa aux représentations propres en parlant d' «intuitions» (Anschauungen). Quelques années après la publication de son premier livre, de plus en plus préoccupé par la logique, il était prêt à élaborer davantage cette distinction.

Dans les écrits de Husserl qui nous intéressent ici, les intuitions sont mises en contraste avec les représentations (Repräsentationen), un nom qu'il emploie souvent pour désigner les représentations impropres. Dès qu'une Repräsentation a lieu dans la conscience, il doit y avoir selon lui une intuition qui a une fonction représentative. Une grande partie de ce que nous considérons relever de la perception est intuitif seulement à un faible degré, et cela à un moment précis ${ }^{23}$. Alors que nous disons voir le tout d'une chose, comme une table ou une pièce de monnaie, nous n'avons dans les faits qu'une intuition momentanée de certaines formes et couleurs qui représentent le tout. En ce qui concerne les mélodies, les changements, et tout ce qui peut être étendu temporellement, Husserl soutient que seul un très court segment temporel peut être accessible à l'intuition ${ }^{24}$. L'aboutissement de ces considérations est que la Repräsentation joue un rôle énorme tout au long des processus conscients que nous appelons habituellement la "perception ». Étant donné que Husserl avait déjà développé le point de vue selon lequel les mathématiques dépendent pour l'essentiel des représentations impropres, il est clair que, pour lui, notre vie consciente repose largement sur les Repräsentationen, dans le domaine tant conceptuel que perceptuel.

Ainsi, il peut sembler que Husserl restreigne le domaine de l'intuition à un strict minimum, mais on doit garder à l'esprit qu'il autorise les représentations de fantaisie (Phantasievorstellungen) à l'intérieur de ce domaine ${ }^{25}$. Il est favorable à la recommandation de Meinong selon laquelle de telles représentations ne devraient pas être appelées des intuitions, mais plutôt des "représentations intuitives" (anschauliche Vorstellungen), car autrement on pourrait penser que les intuitions appartiennent entièrement au domaine perceptuel $^{26}$, bien que Husserl, comme nous le verrons, ne juge pas qu'il soit

22. Pour une étude détaillée du premier ouvrage de Husserl sur la philosophie de l'arithmétique ainsi que des textes en rapport à celle-ci, voir Ierna (2005) et Ierna (2006).

23. Husserliana XXII, pp. 101sq.

24. Husserliana XXII, pp. 269sq.

25. Husserliana XXII, pp. 103sq. En 1898, mais aussi dans des leçons qu'il allait donner quelques années plus tard, Husserl a porté plus d'attention aux représentations de fantaisie. Leur caractérisation de représentations intuitives est restée à la base de sa conception de l'imagination, en opposition à la suggestion de Brentano de considérer qu'elles se rapprochent seulement plus ou moins des représentations propres. Voir Rollinger (1993), ou sa révision dans Rollinger (2008), pp. 29-50.

26. Husserliana XII, p. 104n. Voir Meinong (1888). 
en fin de compte opportun de traiter des intuitions et des Repräsentationen en ajoutant certains termes à Vorstellung. De plus, Husserl constate que le même type de Repräsentation qu'on retrouve dans la perception, et par lequel l'intuition d'un aspect d'une chose représente la chose au complet, se retrouve aussi dans les représentations de fantaisie. Lorsque nous imaginons une chose, nous l'imaginons en fait à partir de certaines perspectives qui ont une fonction représentative.

Eu égard au fait que les intuitions ont été thématisées par Kant tout au long de la Kritik der reinen Vernunft et subséquemment par d'autres philosophes, comme Bolzano ${ }^{27}$, en contraste avec les concepts $(\text { Begriffe })^{28}$, il est important d'insister ici sur le fait que ce contraste n'est pas du tout ce que Husserl avait à l'esprit ${ }^{29}$. De son point de vue, aussi bien les intuitions que les Repräsentationen peuvent être conceptuelles. Dans le concept husserlien de remplissement (Erfüllung), on retrouve une idée étroitement liée à celle-ci ${ }^{30}$. Selon Husserl, une Repräsentation peut se produire avec un certain intérêt à son remplissement. Dans ce cas, la Repräsentation est une intention (Intention). Si on obtient l'intuition correspondante, alors cette intention est remplie. Conséquemment, dans l'entreprise initiale de Husserl d'investiguer la sphère des représentations, l'accent est déjà mis sur la conséquence épistémologique de sa distinction entre intuitions et Repräsentationen.

Même si Husserl abandonnera en fin de compte le concept d'objet immanent à l'acte dans lequel il est visé, il travaille encore avec ce concept dans les textes qui nous intéressent ici. Selon Husserl, les intuitions sont des représentations d'objets immanents à celles-ci, alors que cela n'est pas le cas selon lui en ce qui concerne les Repräsentationen ${ }^{31}$. Toutefois, il croit en même temps qu'on ne peut rien représenter (repräsentieren) autrement qu'au moyen d'objets immanents, c'est-à-dire au moyen des contenus intuitifs. Bien que la conception de Husserl ne soit pas très éloignée de celle de Brentano sur ce point, nous verrons bientôt qu'en 1893 et 1894 sa théorie du jugement diverge déjà considérablement des doctrines de son mentor. Toutefois, la théorie de la représentation de Husserl est à cette époque également

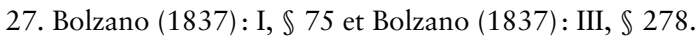

28. Or il y a un autre contraste mis de l'avant dans Kerry $(1885,1886,1887,1889$, 1890 et 1891) entre l'intuition et le processus psychique (psychische Verarbeitung) qui provient de l'école de Brentano et qui est passé presque complètement sous silence. Voir Rollinger (1999), pp. 125-137.

29. Husserliana XXII, p. 104: "Ce que nous nous représentons conceptuellement, nous pouvons souvent le gagner par l'intuition [...]" (Von dem, was wir begrifflich vorstellen, können wir oft durch Anschauung gewinnen [...]). La conception selon laquelle les concepts ne sont pas simplement dérivés génétiquement des intuitions, mais aussi illustrés par celles-ci est non seulement cruciale pour la première philosophie des mathématiques de Husserl telle qu'on la trouve dans Husserliana XII, mais elle est aussi de grande importance pour les développements plus tardifs de sa pensée.

30. Husserliana XXII, p. 296sq.

31. Ibid., p. 110 sq. 
très différente de celle de Brentano. Selon Husserl, ou bien les intuitions et les Repräsentationen sont des représentations en des sens différents ${ }^{32}$ ou alors, si ce n'est pas le cas, il vaut difficilement la peine de les traiter comme une unité spécifique ${ }^{33}$, alors que Brentano concevait les représentations comme appartenant à une seule classe unitaire de phénomènes mentaux.

\section{Jugements}

Penchons-nous maintenant sur la théorie du jugement développée dans Versuch et dans Eigentliche und uneigentliche Urteile. Tout comme Brentano, Husserl conçoit les jugements comme des actes de conscience se référant à des objets, sauf lorsqu'il parle explicitement des «jugements objectifs» (objektive Urteile) dont nous discuterons plus loin. De plus, bien qu'il n'adhère pas explicitement à la doctrine de Brentano selon laquelle ces actes sont dans tous les cas des instances d'acceptation ou de rejet exprimés idéalement par des énoncés existentiels, le point de départ des considérations de Husserl est à tout le moins les jugements exprimés de cette manière. Cela mis à part, les doctrines brentaniennes ne jouent pas un rôle important dans la première théorie du jugement de Husserl. Comme nous le verrons ici, il critique d'ailleurs explicitement une de ces doctrines dans Eigentliche und uneigentliche Urteile, et d'autres doctrines semblent aussi incompatibles avec sa théorie du jugement.

Nous exposerons maintenant les aspects suivants de cette théorie: 1) les jugements en relation aux représentations; 2) les assomptions comme actes correspondant aux jugements; 3) les jugements objectifs; 4) les jugements impropres; 5) les états de choses; et 6) les jugements et les formations catégoriales.

\subsection{Jugements et représentations}

Que les actes de conscience qui ne sont pas des représentations soient basés sur des représentations, c'est là une thèse répandue de la psychologie descriptive de Brentano. Celui-ci distingue les jugements des représentations et considère que les premiers sont des actes fondés sur les secondes. La conception qui en résulte, selon laquelle les jugements sont basés sur des représentations, est précisément celle que Husserl rejette dans Eigentliche und uneigentliche Urteile.

Husserl confronte Brentano sur ce point lorsqu'il soulève la question de savoir ce qui distingue l'acceptation de quelque chose (juger affirmative-

32. Ibid., p. 283.

33. Husserliana XXII, p. 119. Pour cette raison, Husserl préfère parler d'intuitions et de Repräsentationen plutôt que d'utiliser "des termes [...] qui sont constitués à partir du terme "représentation" ", tels que "représentation propre " (eigentliche Vorstellung) et "représentation impropre» (uneigentliche Vorstellung). 
ment que cela existe) et l'imagination de quelque chose. Il fait l'affirmation suivante dans ce contexte:

Or Brentano pourrait dire ici: dans le premier cas, nous représentons simplement, et dans le second nous jugeons en plus. Mais là, je dirais: 1) d'abord je ne trouve pas dans le deuxième [cas] de dédoublement, à moins que [l'acte d'] appréhender, de remarquer, soit appelé un «représenter». 2) Ce qui s'ajoute dans le deuxième cas, lorsque je laisse de côté le jugement explicite «il existe », ne se place pas devant moi comme un acte, mais plutôt comme une qualité interne dirigée sur le contenu, qui est toutefois dirigée sur le sujet de manière similaire aux sentiments répandus, etc., sur l'objet; et cela peut être quelque chose d'ultérieur. En tout cas, il en va autrement avec de telles qualités qu'avec quelque chose de jugé, de voulu, de désiré, d'espéré, etc. 3) La qualité de l'être, c'est ainsi que je l'appellerais, ne se maintient pas simplement pendant un point temporel fugace; elle se maintient de manière continue. Or on pourrait dire que l'[acte de] remarquer en fait autant, que c'est un acte qui perdure, et que cela vaut tout autant pour l'[acte de] juger qui est fusionné avec l'[acte de] saisir $^{34}$.

Conséquemment, Husserl utilise la notion de «qualité de l'être » pour apporter une autre solution que celle de Brentano. Bien entendu, ce n'est pas du tout la manière par laquelle il traitera plus tard de ce problème dans les Recherches logiques, alors qu'il mettra de l'avant la distinction entre représentations positionnelles et représentations non positionnelles ${ }^{35}$. Néanmoins, le rejet de la thèse de Brentano, selon laquelle un jugement est basé sur une représentation, est pleinement affirmé dans le passage que nous venons de citer $^{36}$.

Il importe également de mentionner que, pour Brentano, il y a jugement dès qu'il y a perception au sens ordinaire du terme, alors que Husserl affirme la chose suivante à propos des jugements :

Par exemple, je juge: «le four est noir». J'affirme, et la chose est faite. Cela ressemble à la décision, qui ne fait que s'accomplir et qui se transforme ensuite en une disposition s'actualisant ad libitum. L['acte de] désirer et l['acte d'] aimer peuvent en effet se manifester en actes individuellement achevés et pourtant persister en tant que phénomènes familiers. Dans l'[acte de] juger, on devrait donc aussi distinguer entre un juger familier et un acte de jugement particulier $^{37}$.

34. Husserliana XL, p. 42.

35. Husserliana XIX/1, pp. 505sq.

36. Voir aussi le passage suivant du quatrième appendice à Eigentliche und uneigentliche Urteile: "C'est d'abord au moyen du juger faux que nous en venons à la distinction entre la simple représentation et l'être-réel. Le jugement est suspendu et ne subsiste alors de lui que le simple représenter, comme jugement suspendu qui enclave la pensée dans le jugement. [...] Ainsi, d'un point de vue génétique, le jugement vient avant la "simple représentation", et cette dernière est une modification psychologique du jugement "(Husserliana XL, p. 57).

37. Husserliana XL, p. 42. 
Ainsi, là où Brentano voit des actes, Husserl voit une abondance de dispositions, et c'est seulement dans des cas particuliers qu'il voit là des actes au sens propre du terme. Alors que, pour Brentano, la perception est un acte de jugement, pour Husserl, c'est apparemment le représenter d'objets qui ont la propriété d'être, et c'est seulement de manière occasionnelle et fugace que la perception est un juger analogue à une décision.

\subsection{Jugements et assomptions}

Alors que Brentano soutenait qu'il n'y a que trois classes d'actes de conscience, Husserl remet apparemment cette classification en question (du moins de manière implicite) en parlant constamment d'une classe additionnelle de tels actes, à savoir les "assomptions ${ }^{38}$. De tels actes sont d'une grande importance pour la théorie du jugement parce qu'ils sont également exprimés dans des énoncés, comme le sont les jugements, et parce qu'ils interviennent dans l'émission de certains jugements, à savoir les jugements "sous assomption» (unter Assumption) tels que notre jugement que Jupiter est le plus grand des dieux olympiens.

Chez le premier Husserl, cette notion de juger sous assomption nous est bien sûr familière depuis la publication de son manuscrit de 1894 sur les objets intentionnels (Intentionale Gegenstände) ${ }^{39}$. Peu après la publication de Über Annabmen de Meinong en $1902^{40}$, Husserl envoya ce manuscrit à l'auteur du livre, mais il lui envoya également le manuscrit de Versuch ${ }^{41}$. La raison apparente à cela est à trouver dans le fait que Husserl avait déjà découvert le domaine des assomptions longtemps avant Meinong ou quiconque parmi ses étudiants ${ }^{42}$.

Le passage suivant de Versuch, dans lequel Husserl parle des assomptions, est ici d'un intérêt particulier:

La position hypothétique, l'[acte d'] «assumer », est un comportement psychique particulier face à l'état de choses placé («représenté») objectivement et relève de la même irréductibilité psychologique que l'[acte de] reconnaître, de

38. Bien que Husserl emploie parfois le terme "Annahmen" en référence à de tels actes, il emploie aussi parfois ce terme pour indiquer une acceptation, c'est-à-dire un jugement affirmatif.

39. Voir Husserliana XXII, pp. 303-348. Une édition améliorée de ce texte a été publiée dans Schuhmann (dir.) (1990-1991), pp. 137-176.

40. Voir Meinong (1902). Pour une comparaison entre la théorie meinongienne des assomptions et celle de Husserl dans les Recherches logiques, voir Rollinger (1999), pp. 186199.

41. Cela est indiqué dans une lettre de Husserl à Meinong datée du 5 avril 1902. Voir Schuhmann (dir.) (1994), I, pp. 139-145.

42. Meinong a répondu à Husserl par une lettre, datée du 10 avril 1902 (voir Schuhmann (dir.), pp. 145-147), dans laquelle il refuse la demande que celui-ci lui a faite de lire ses manuscrits, étant à ce moment préoccupé par d'autres problèmes liés à ses leçons, mais aussi parce qu'il trouvait Husserl beaucoup trop sensible quant à la protection de ses possessions intellectuelles. 
rejeter, de présumer, de douter, etc. De la réflexion sur ce comportement psychique résulte la paire corrélative des concepts de [l'acte d']assumer et de ce qui est assumé, et de ce dernier concept, par abstraction toutefois de l'individuel et du subjectif, résulte le concept d'objet d'assomption, d'hypothèse ${ }^{43}$.

Conséquemment, l'analogie entre les jugements et les assomptions est valide non seulement pour la sphère subjective des actes, mais aussi pour la sphère objective correspondante.

\subsection{Jugements objectifs}

Dans Versuch, Husserl aborde la notion de «jugements objectifs » ${ }^{44}$, pour laquelle il emploie également le terme de "jugement en soi » (Urteil an sich) ${ }^{45}$ qui suggère une influence de Bolzano ${ }^{46}$. Toutefois, Husserl parle de ces jugements également en termes de «jugements objectivés» (objektivierte Urteile $)^{47}$ et soulève la question de savoir comment une telle objectivation (Objektivierung) a lieu ${ }^{48}$. Conséquemment, les jugements objectifs sont en quelque sorte des produits. Ainsi, dans Versuch, Husserl n'est pas encore arrivé à son concept d'objets idéaux comme thème de la logique pure. Les jugements sont objectivés dans la mesure où le "moment d'évidente vérité ou fausseté » (das Moment der evidenten Wabrheit oder Falschbeit) est "souligné abstractivement et saisi conceptuellement " (abstraktiv hervorgehoben und begrifflich gefasst), un processus qui de plus implique pour lui la représentation du jugement au moyen de la forme linguistique d'un énoncé (Aussage $)^{49}$. Alors que Husserl parle de l'entité logique idéale (das ideale logische Wesen) dans ses considérations sur l'objectivation, il utilise ici le terme "idéal » en un sens normatif ${ }^{50}$. L'entité en question n'a rien à voir avec une proposition, mais est plutôt un esprit parfait dont l'évidence est à son degré le plus complet ${ }^{51}$.

43. Husserliana XL, pp. 18 sq.

44. Ibid., pp. 16, 18 et 29.

45. Ibid., p. 29.

46. Alors que Bolzano parle habituellement de propositions en soi (Sätze an sich) plutôt que de jugements en soi, il soutient que leur relation aux jugements est la même que celle des représentations objectives aux représentations subjectives.

47. Husserliana XL, p. 6.

48. Ibid., p. $26 s q$.

49. Husserliana XL, p. 29.

50. Husserliana XL, pp. 29sq. Dans les Recherches logiques, Husserl souligne explicitement que l'idéalité qu'il attribue aux propositions et aux autres significations n'est pas à concevoir normativement. Voir Husserliana XIX/1, p. 107.

51. Cette notion intervient chez Sigwart (1889), p. 233sq. ainsi qu'à d'autres endroits du même ouvrage. Bien que Husserl n'ait certainement pas emprunté sa notion d'esprit idéal à cet ouvrage, l'application de celle-ci dans le contexte de la logique et de l'épistémologie suggère une influence de Sigwart sur Husserl. Voir également le début de Versuch ainsi que celui d'Eigentliche und uneigentliche Urteile (Husserliana XL, p. 1 et pp. 31sq.), où Sigwart, comme Brentano, s'avère être l'interlocuteur de Husserl dans la formulation des problèmes auxquels il tente de répondre. Une étude approfondie de la relation entre Husserl et Sigwart, dans laquelle 
Le concept d'une telle entité nous permet de former le concept d'une évidence objective correspondant à la notion de vérité objective.

\subsection{Jugements impropres}

Alors que Husserl travaille à son concept de jugement objectif dans Versuch, il est fort préoccupé, dans Eigentliche und uneigentliche Urteile, par un sujet qui concerne le versant subjectif de la théorie du jugement. Comme nous avons vu qu'il distinguait entre les représentations propres et les représentations impropres (ou encore, entre les intuitions et les Repräsentationen), il fait une distinction correspondante entre les jugements propres et les jugements impropres. La plus grande partie de nos jugements, nous dit Husserl, est en fait constituée de jugements impropres, puisque nous pouvons juger, par exemple, qu'il y a un feu à un endroit situé au-delà de notre champ de perception $^{52}$.

La distinction entre ces deux types de jugements n'était cependant pas inconnue de Brentano. Dans un de ses cours de logique, il affirme:

Comme nous l'avons déjà remarqué, le jugement présuppose une représentation, et les différences dans le mode du représenter sont donc aussi significatives pour les jugements. Tout ce qui a été déterminé plus tôt est valide ici. Il y a donc par exemple des jugements dont les objets sont représentés de manière impropre $^{53}$.

Ici, on voit bien sûr que Brentano parle de jugements qui sont fondés sur des représentations impropres. Évidemment, Husserl aurait pu au départ avoir à l'esprit le même concept de jugement impropre. Il ne peut toutefois se permettre une caractérisation comme celle de Brentano, à savoir que le jugement est fondé sur une représentation impropre, puisqu'il rejette la thèse qu'un jugement est fondé sur une représentation. Dans Eigentliche und uneigentliche Urteile, la seule option à la portée de Husserl pour distinguer entre ces deux types de jugements serait de recourir à la notion d'objet immanent. Puisque les représentations propres diffèrent des représentations impropres dans la mesure où les objets de ces premières sont immanents à ces dernières, on pourrait dire la même chose des jugements propres en contraste avec les jugements impropres, bien que Husserl ne formule pas explicitement un tel critère ${ }^{54}$.

serait pris en considération le matériel pertinent, publié et non publié, constituerait sans l'ombre d'un doute une contribution substantielle à la compréhension du développement de la pensée de Husserl.

52. Husserliana XL, p. 34sq.

53. Brentano EL 80/13243.

54. La seule mention d'un objet immanent dans Eigentliche und uneigentliche Urteile se trouve dans Husserliana XL, p. 49, où il est dit qu'un tel objet serait un état de choses, ce dont nous discuterons plus loin. 


\section{5 États de choses}

Depuis les Recherches logiques et d'autres écrits plus tardifs de Husserl, nous nous sommes bien entendu familiarisés avec la notion d'état de choses (Sachverhalt). Nous savons également que, pour Husserl, le point de départ de cet usage se trouve dans les leçons de logique de Carl Stumpf, en 1888, dans lesquelles l'être de Dieu est donné comme exemple d'un état de choses en relation au jugement que Dieu est, et où le non-être de Dieu est donné comme exemple d'un état de choses en relation au jugement que Dieu n'est pas. De plus, pour Stumpf, les états de choses sont qualifiés de contenus des jugements en question ${ }^{55}$.

Dans Eigentliche und uneigentliche Urteile, Husserl s'essaie à deux différentes caractérisations des états de choses. Il soulève d'abord la question de savoir si ce sont des jugements représentés ${ }^{56}$. Bien que cette question puisse nous paraître étrange, la notion de "jugement représenté » (vorgestelltes Urteil) est présente dans la littérature avec laquelle Husserl était fami$\operatorname{lier}^{57}$. De plus, si nous considérons les états de choses comme étant formulés par des subordonnées complétives, par exemple que l'or est jaune, il n'est pas besoin d'aller chercher bien loin pour conclure que lorsque nous pensons à ce à quoi réfèrent de telles subordonnées complétives, nous nous représentons des jugements, bien que de manière indéterminée (eu égard à celui qui émet le jugement, au moment de son émission, etc.) Bien que Husserl ait initialement répondu de manière affirmative à la question de savoir si les jugements et les états de choses représentés sont la même chose, il raye sa réponse affirmative et développe une autre caractérisation des états de choses ${ }^{58}$.

Apparemment, cette caractérisation est très proche de celle de Stumpf. Selon cette conception, un état de choses est "en fait seulement un abstractum. C'est quelque chose de commun à plusieurs phénomènes " ${ }^{59}$. Ici, l'emploi du terme Abstraktum par Husserl signifie simplement qu'un état de choses est une partie dépendante (unselbständiger Teil) de ces phénomènes différents ${ }^{60}$. On peut juger que quelque chose est le cas, mais on peut aussi,

55. Rollinger (1999), p. 313.

56. Husserliana XL, p. 48 sq.

57. Voir Meinong (1882), p. 105sq., Sigwart (1889), p. 232 et 304, ainsi que Marty (1884), p. 179 et Marty (1894), p. 468.

58. Husserliana XL, p. 45n. Cf. le deuxième appendice à Eigentliche und uneigentliche Urteile, pp. 53, où Husserl utilise cette caractérisation des états de choses en réflexions non conclusives sur le fait de savoir s'ils sont formés par le langage. Si nous considérons toutefois qu'un état de choses peut être nommé par une subordonnée complétive, par exemple «que Dieu existe » ou "que Dieu n'existe pas », il n'est pas exagéré de suggérer que lorsque nous nous représentons des objets nommés par de telles subordonnées, nous nous représentons en fait des jugements, par exemple le jugement que Dieu existe ou le jugement que Dieu n'existe pas.

59. Husserliana XL, p. 49.

60. Husserliana XXII, p. 92 sq. 
par exemple, assumer que c'est le cas où on peut souhaiter que ce le soit. Ici, l'état de choses peut être commun à tous ces phénomènes, comme Husserl le conçoit dans Eigentliche und uneigentliche Urteile, mais non pas comme un objet qui existe indépendamment d'eux ${ }^{61}$. Bien qu'il n'explique pas tout à fait ce qui motive ce changement dans sa caractérisation des états de choses, on peut s'apercevoir qu'il serait difficile de les considérer comme des jugements représentés qui sont aussi le corrélat d'assomptions ou de souhaits assomptifs, puisque cela impliquerait de complexifier la description de ces derniers phénomènes, comme si on ne pouvait pas souhaiter que quelque chose soit le cas sans également se représenter le jugement que c'est le cas.

Dans Versuch, Husserl parle toutefois d'états de choses comme de ce qu'on accepte ou rejette dans nos jugements ${ }^{62}$ et il dit que ce sont ceux-ci, et non nos jugements, qui font figure de porteurs des modalités comme la nécessité et l'impossibilité ${ }^{63}$. De plus, à titre de confirmation du statut objectif de ceux-ci, notons son affirmation selon laquelle, lorsque nous parlons de faits (Tatsachen),

nous ne visons pas par là l'état de choses subjectif, psychologique, mais plutôt l'état de choses objectif; nous pensons que ce n'est pas seulement à nous que manque la raison conceptuelle évidente, mais qu'elle manque aussi «en soi », comme par exemple lorsque nous désignons le jugement évident «Ceci (que je me représente maintenant) est un rouge» comme un jugement simplement factuel, sa matière comme un fait évident ${ }^{64}$.

De plus, il dit à propos de l'évidence de la relation d'entraînement $(A b f o l g e)$ : "Il ne s'agit pas de la simple appréhension du fait subjectif interne, du vécu hic et nunc, mais plutôt de l'évidence que les deux états de

61. Dans la terminologie des Recherches logiques, cela fera de l'état de choses quelque chose de très apparenté à la matière d'un acte de conscience en contraste avec sa qualité. Voir Husserliana XIX/1, p. 425-431. Cela semble entrer en conflit avec le deuxième appendice de Eigentliche und uneigentliche Urteile, où Husserl affirme "On peut aussi séparer le concept d'état de choses de celui de matière de jugement " (Den Begriff des Sachverhalts kann man auch von dem der Urteilsmaterie trennen) (Husserliana XL, p. 55). Ici, toutefois, il vise spécifiquement la matière du jugement et non celle de l'acte comme tel, tel qu'il le conçoit dans les Recherches logiques. De plus, dans l'appendice que nous venons d'évoquer, Husserl se satisfait de caractériser les états de choses comme des jugements présentés, comme nous venons de le mentionner, alors qu'il se penche sur une autre caractérisation de ceux-ci dans Eigentliche und uneigentliche Urteile.

62. Husserliana XL, p. 6sq. Toutefois, en même temps, Husserl parle ici d'états de choses mutuellement exclusifs. La question de savoir comment un état de choses peut en exclure un autre - puisque dans ce cas l'état de choses exclu ne subsiste pas du tout et n'est donc pas du tout un état de choses - deviendra plus tard un problème pour son ontologie formelle. Voir Husserliana XL, p. 162-167, dans lequel Husserl tente de résoudre ce problème.

63. Husserliana XL, p. 5. Cf. Husserliana XL, p. 14: «Là où un jugement découle d'un autre et ne fait simplement que lui succéder temporellement, on peut et on doit souvent caractériser de nécessaire l'état de choses qui en est déduit. »

64. Husserliana XL, p. 12. 
choses sont dans une relation objective d'entraînement ${ }^{65}$. En ce sens, les états de choses ne sont aucunement conçus comme des parties dépendantes des jugements et d'autres actes de conscience.

Le concept d'état de choses, tel qu'il est utilisé dans Versuch, est plus près de celui que Husserl développera plus tard dans les années 1890, concept qui fera sa première apparition publique dans les Recherches logiques ${ }^{66}$. Bien que cela puisse être considéré comme une indication que Versuch fut écrit plus tard que Eigentliche und uneigentliche Urteile, une telle conclusion doit être prise avec un grain de sel. Comme on peut le voir tout au long des textes de Husserl publiés récemment sur la théorie du jugement, le concept d'état de choses est fluctuant. Nous savons que, dans sa Bedeutungslehre de 1908, Husserl recommandait l'utilisation différenciée des termes Sachlage et Sachverhalt $t^{67}$. Toutefois, cette terminologie n'a pas été rigoureusement maintenue ${ }^{68}$. Conséquemment, il n'est pas du tout improbable que Husserl utilisât le même terme dans les années 1893 et 1894 afin d'indiquer parfois quelque chose "d'objectif» (ou de transcendant à la conscience), parfois quelque chose de «subjectif» (ou d'immanent à la conscience).

\subsection{Jugements et formations catégoriales}

Bien entendu, plusieurs écrits de Husserl nous ont familiarisé avec le problème de la formation catégoriale tel qu'il se manifeste dans diverses structures syntaxiques du langage. Toutefois, il vaut la peine d'insister sur le fait que, dès l'époque d'Eigentliche und uneigentliche Urteile, cette question le préoccupait déjà. Pour être bref, le problème consiste à savoir si des structures telles que la prédication (par exemple «le four est noir») ou l'attribution ( «le four qui est noir») sont purement linguistiques par nature où si elles émergent d'une sorte d'activité mentale. Une autre option extrêmement réaliste serait que les structures en question soient considérées comme reflétant simplement les choses telles qu'elles sont dans le monde, même si Husserl n'accorde pratiquement aucune considération à cette option. Bien que dans sa première théorie du jugement la plupart de ses méditations sur la formation catégoriale soient aporétiques - sans compter que leur traitement prendrait ici beaucoup trop d'espace ${ }^{69}-$, il est néanmoins pertinent de noter qu'elles constituent déjà un aspect important de la première théorie du jugement de Husserl.

65. Husserliana XL, p. 16.

66. Voir Husserliana XIX/1, pp. 32 ff., 44, 84, 99, 118, 167, 186, 381, 416sq. Voir également le texte de la fin des années 1890 publié dans Husserliana XL, pp. 82-91, ainsi que ses appendices (pp. 91-139). Dans ces textes, non seulement le terme Satz est utilisé fréquemment, mais Husserl est très préoccupé par la distinction entre Satz et état de choses. C'est là un problème qui n'est pas encore thématisé dans Versuch.

67. Voir Husserliana XXVI, p. 29 sq.

68. Voir particulièrement les textes publiés dans Husserliana XL, p. 141-162 et 176-208.

69. Voir Husserliana XL, pp. 53sq., 59sq., 64sq. 


\section{Remarques finales}

Lorsqu'un philosophe a autant d'influence que Husserl, il est tout simplement naturel d'examiner les différentes phases du développement de sa pensée. Déjà pour cette raison, les aspects de sa première théorie du jugement qui ont été présentés ici méritent notre attention. Tout comme le Kant de la phase pré-critique représente un point d'intérêt pour les chercheurs, il en va de même du Husserl de la phase pré-transcendantale. D'un autre point de vue, cette phase du développement de Husserl devient cependant encore plus intéressante vue de la perspective récemment présentée comme étant celle de la phénoménologie autrichienne ${ }^{70}$. Alors que Husserl en est venu plus tard à soutenir le point de vue selon lequel la phénoménologie possède une méthode particulière, méthode dont la formulation et la reformulation ad nauseam ne l'a jamais lassé, la phénoménologie autrichienne opère à partir de la thèse brentanienne selon laquelle la vraie méthode de la philosophie n'est rien d'autre que celle des sciences naturelles. De plus, la phénoménologie autrichienne est d'abord et avant tout une description de la conscience dirigée intentionnellement, et ensuite une théorie des objets corrélatifs à la conscience. Les deux textes qui ont été discutés ici illustrent de manière frappante cette double nature de la phénoménologie autrichienne. Alors que Eigentliche und uneigentliche Urteile est en premier lieu une description de la conscience judicative, Versuch est davantage centré sur l'aspect objectif des jugements. Nous avons là une confirmation supplémentaire que le jeune Husserl était un phénoménologue autrichien. Dans ses premiers écrits, on est en effet souvent confronté à des positions qui sont affirmées de manière plus tranchée que dans ses textes tardifs ainsi qu'à des problèmes qui sont «résolus » dans les années subséquentes, et cela crée indubitablement l'impression que ces premiers écrits constituent surtout des tâtonnements vers des positions plus mûres. Évidemment, cela peut être vrai jusqu'à un certain point et dans certains cas (par exemple en ce qui concerne le problème des formations catégoriales), mais il faut insister sur le fait que Husserl accapare plus tard un fardeau méthodologique qui est fréquemment un handicap à ces positions soi-disant mûres. Alors que ceux qui sont intéressés d'abord et avant tout au dernier Husserl, c'est-à-dire à Husserl le Transcendantaliste allemand, vont considérer cette première théorie du jugement comme une simple phase de transition à noter dans sa biographie intellectuelle et ainsi comme un élément d'intérêt simplement marginal, nous voulons y voir là, dans le contexte de la phénoménologie autrichienne, un point qui mérite une attention et un respect considérablement plus grands.

Traduit de l'anglais par Guillaume Fréchette

70. Rollinger (2008), p. 1-27. 


\section{Bibliographie}

Bergmann, Julius. 1895. Die Grundprobleme der Logik, Berlin, Ernst Siegfried Müller und Sohn.

Bolzano, Bernard. 1837. Wissenschaftslehre. 4 vol., Sulzbach, Seidel.

Brentano, Franz. 1874. Psychologie vom empirischen Standpunkte, Leipzig, Duncker \& Humblot.

Brentano, Franz, Mayer-Hildebrand (dir.). Die Lehre vom richtigen Urteil, Bern, Francke, 1956.

Brentano, Franz (EL 72). Die elementare Logik und die in ihr nötigen Reformen. Notes de cours non publiées de 1884-1885. Harvard, Houghton Library.

Brentano, Franz (EL 80). Logik. Notes de cours non publiées (sans date). Harvard, Houghton Library.

Husserl, Edmund. Landgrebe (dir.). Erfahrung und Urteil. Untersuchungen zur Genealogie der Logik, Hamburg, Claasen \& Goverts, 1948.

Husserl, Edmund. Lothar Eley (dir.), Husserliana XII: Philosophie der Arithmetik. Mit ergänzenden Texten (1890-1901), La Haye, Martinus Nijhoff, 1970.

Husserl, Edmund. Bernhard Rang (dir.), Husserliana XXII: Aufsätze und Rezensionen (1890-1910), La Haye, Martinus Nijhoff, 1979.

Husserl, Edmund. Husserliana XIX/1: Logische Untersuchungen. Zweiter Band, 1. Teil. Untersuchung zur Phänomenologie und Theorie der Erkenntnis, La Haye, Martinus Nijhoff, 1984.

- Husserliana XIX/2: Logische Untersuchungen. Zweiter Band, 2. Teil. Untersuchung zur Phänomenologie und Theorie der Erkenntnis, La Haye, Martinus Nijhoff, 1984.

Husserl, Edmund. Robin D. Rollinger (dir.), Husserliana XL: Untersuchungen zur Urteilstheorie: Texte aus dem Nachlass (1893-1918), Dordrecht, Springer, 2009.

Jacquette, Dale (dir.). The Cambridge Companion to Brentano, Cambridge, Cambridge University Press, 2004.

Kant, Immanuel. Wilhelm Weischedel (dir.). Kritik der reinen Vernunft, 2 vol., Frankfurt am Main, Suhrkamp, 1974.

Kerry, Benno. 1885. «Über Anschauung und ihre psychische Verarbeitung ", Vierteljahrsschrift für wissenschaftliche Philosophie 9, pp. 433-493, $1^{\mathrm{er}}$ article.

—. 1886. "Über Anschauung und ihre psychische Verarbeitung », Vierteljahrsschrift für wissenschaftliche Philosophie 10, pp. 419-467, $2^{\mathrm{e}}$ article.

_. 1887. «Über Anschauung und ihre psychische Verarbeitung », Vierteljahrsschrift für wissenschaftliche Philosophie 11, pp. 53-116, 249-307, $3^{\mathrm{e}}$ et $4^{\mathrm{e}}$ article.

- 1889. «Über Anschauung und ihre psychische Verarbeitung », Vierteljahrsschrift für wissenschaftliche Philosophie 13, pp. 71-124, 392-419, $5^{\mathrm{e}}$ et $6^{\mathrm{e}}$ article.

—. 1890. "Über Anschauung und ihre psychische Verarbeitung ", Vierteljahrsschrift für wissenschaftliche Philosophie 14, pp. 317-353.

—. 1891. «Über Anschauung und ihre psychische Verarbeitung », Vierteljabrsschrift für wissenschaftliche Philosophie 15, pp. 127-167.

Ierna, Carlo. "The Beginnings of Husserl's Philosophy, Part 1: From Über den Begriff der Zahl to Philosophie der Arithmetik ", The New Yearbook for Phenomenology and Phenomenological Philosophy 5, 2005, pp. 1-56. 
- « The Beginnings of Husserl's Philosophy, Part 2: Philosophical and Mathematical Background ", The New Yearbook for Phenomenology and Phenomenological Philosophy 6, 2006, pp. 23-71.

Lotze, Hermann. 1880. Logik. Drei Bücher vom Denken, vom Untersuchen und vom Erkennen, Leipzig, Hizel, $2^{\mathrm{e}}$ édition.

Marty, Anton. 1884. «Über subjektlose Sätze », Vierteljahrsschrift für wissenschaftliche Philosophie 8, pp. 56-94, 161-192, 292-340, $1^{\mathrm{er}}, 2^{\mathrm{e}}$ et $3^{\mathrm{e}}$ article.

—. 1894. "Über subjektlose Sätze », Vierteljahrsschrift für wissenschaftliche Philosophie 18, pp. 320-356, 421-471, $4^{\mathrm{e}}$ et $5^{\mathrm{e}}$ article.

—_ 1895. "Über subjektlose Sätze », Vierteljahrsschrift für wissenschaftliche Philosophie 19, pp. 19-87, 263-334.

Meinong, Alexius. 1877. Hume-Studien I: Zur Geschichte und Kritik der modernen Nominalismus, Vienna, Kaiserliche Akademie der Wissenschaften.

- 1882. Hume-Studien II : Zur Relationstheorie, Vienna, Kaiserliche Akademie der Wissenschaften.

- 1888. "Phantasie-Vorstellung und Phantasie", Zeitschrift für Philosophie und philosophische Kritik 95, pp. 161-244.

—. 1902. Über Annahmen, Leipzig, Johann Ambrosius Barth.

Rollinger, Robin D. "Husserl and Brentano on Imagination », Archiv für Geschichte der Philosophie 75, 1993, pp. 195-210.

- Husserl's Position in the School of Brentano, Dordrecht/Boston/London, Kluwer, 1999.

—. «Husserl's Elementary Logic: His 1896 Logic in its Nineteenth Century Context », Studia Phaenomenologica. Romanian Journal of Phenomenology 3, 2003, pp. 195-214.

_. «Brentano and Husserl», in Jacquette (dir.), 2004a, pp. 255-276.

—. "Austrian Theories of Judgment: Bolzano, Brentano, Meinong, and Husserl », in Chrudzimski and Huemer, 2004b, pp. 257-284.

- Austrian Phenomenology: Brentano, Husserl, Meinong, and Others on Mind and Object, Frankfurt, Ontos-Verlag, 2008.

- (à paraître) Philosophy of Language and Other Matters in the Philosophical Work of Anton Marty, Amsterdam/Atlanta, Rodopi.

Schuhmann, Karl (dir.). "Husserls Abhandlung "Intentionale Gegenstände”. Edition der ursprünglichen Druckfassung ", Brentano-Studien 3, 1990-1991, pp. 137-176.

—. En collaboration avec Elisabeth Schuhmann. Edmund Husserl. Briefwechsel, 10 vol., Dordrecht/Boston/London, Kluwer, 1994.

—. Cees Leijenhorst et Piet Steenbakkers. Selected Papers on Phenomenology, Dordrecht, Springer, 2004.

Sigwart, Christoph. 1889. Logik. Erster Band: Die Lehre vom Urteil, vom Begriff und vom Schluss, Freiburg i. B., J. C. B. Mohr (Paul Siebeck), $2^{\mathrm{e}}$ édition.

- 1893. Logik. Zweiter Band. Die Methodenlehre. Freiburg i. B., J. C. B. Mohr (Paul Siebeck), $2^{\mathrm{e}}$ edition.

Stumpf, Carl. 1873. Über den psychologischen Ursprung der Raumvorstellung, Leibzig, S. Hirzel.

Twardowski, Kasimir, 1894. Zur Lehre vom Inhalt und Gegenstand der Vorstellungen. Eine psychologische Studie, Vienna, Hölder. 
Wundt, Wilhelm, 1874, Grundzüge der physiologischen Psychologie, 2 vol., Leipzig, Wilhelm Engelmann.

- . 1893. Logik. Die Untersuchung der Principien der Erkenntnis und der Methoden wissenschaftlicher Forschung. Erster Band. Erkenntnislehre, Stuttgart, Ferdinand Enke, $2^{\mathrm{e}}$ édition.

1894. Logik. Die Untersuchung der Principien der Erkenntnis und der Methoden wissenschaftlicher Forschung. Zweiter Band. Methodenlehre, Stuttgart, Ferdinand Enke, $2^{\mathrm{e}}$ édition. 